\title{
Aspiration pneumonia and dysphagia after technically successful repair of oesophageal atresia
}

\author{
A. R. CHR ISPIN, G. W. FRIEDLAND, AND D. J. WA TERSTON \\ From the Hospital for Sick Children, Great Ormond Street, London, W.C.I
}

In the past decade increasingly satisfactory results have been achieved in the surgical repair of the common congenital abnormality of oesophageal atresia with fistula. However, dysphagia and aspiration pneumonia can occur after operation and may well be related to a stricture at the site of the anastomosis or to a recurrent fistula. In some patients, however, no stricture or fistula can be found to account for an episode or episodes of aspiration pneumonia and dysphagia. Fourteen such patients with severe symptoms have been investigated. All had previously had a technically successful one-stage repair of the common type of oesophageal atresia and fistula (Waterston, Bonham-Carter, and Aberdeen, 1963). Radiological studies have revealed abnormalities of oesophageal flow which can account for these symptoms. An understanding of the underlying oesophageal abnormality enables the clinician to make a logical approach to the management of these patients' problems.

\section{CLINICAL DATA}

In these 14 patients (Table I) a primary anastomosis of the blind upper pouch to the lower oesophageal segment was achieved, the fistulous communication with the trachea being closed at the same operation. Six of the 14 patients developed a stricture or diaphragm in the immediate post-operative period, but all were satisfactorily dilated. Later, at oesophagoscopy, three of the six were thought to have a mild narrowing insufficient in itself to cause symptoms. The other three (making 11 in all) had a normal lumen.

The clinical features that prompted radiological investigation were aspiration pneumonia and dysphagia. During infancy a common sequence was an attack of choking and vomiting or restlessness at night followed by or associated with cyanosis or even white asphyxia and cardiac arrest and later pneumonia. Young infants also experienced difficulty with milk feeds, and this was sometimes ameliorated by thickening the feeds. Older infants and children experienced choking and discomfort when swallowing solid lumpy foods. Peas seemed particularly liable to cause this. In one patient a peanut obstructed the lower oesophagus and had to be removed at oesophagoscopy. All patients had a characteristic cough.

\section{RADIOLOGICAL INVESTIGATION}

OESOPHAGUS Cinefluorographic studies were performed on eight patients. In five a recurrence of fistula was initially thought to be a possibility, and such a fistula was excluded by radiological and endoscopic examinations. Ordinarily cinefluorographic studies were carried out with the patient swallowing normally. Usually the patient was examined supine with either a horizontal or vertical beam. Occasionally the patient was also examined in the erect position.

Conventional fluoroscopy and spot filming was carried out in 10 patients, usually with the patient supine but sometimes with the patient erect.

CHEST All patients had chest radiographs. Some patients required many because of recurrent pneumonia.

\section{RADIOLOGICAL FINDINGS}

CONTRAST STUDIES OF OESOPHAGUS Normally, in infants and children lying in the recumbent position and following pharyngeal deglutition, a stripping wave passes down the oesophagus as far as the vestibule, which lies immediately above the stomach. The oesophagus is completely emptied of contrast medium by the stripping wave. Contrast medium passes through the vestibule into the stomach, and, as flow through the vestibule ceases, its lumen closes. 
T A B LE I

\begin{tabular}{|c|c|c|c|c|}
\hline Patient & $\begin{array}{c}\text { Age at } \\
\text { Investigation } \\
\text { (yrs.) }\end{array}$ & $\begin{array}{l}\text { Symptoms Prompting Radiological } \\
\text { Investigation }\end{array}$ & $\begin{array}{l}\text { Radiological } \\
\text { Technique }\end{array}$ & Results of Contrast Studies \\
\hline 1 & $1 \cdot 5$ & $\begin{array}{l}\text { Episodes of choking associated with } \\
\text { cyanosis, rapid respiration, and res- } \\
\text { piratory infections }\end{array}$ & $\begin{array}{l}\text { Fluoroscopy } \\
\text { Cinefluorography }\end{array}$ & $\begin{array}{l}\text { Inefficient stripping action; hiatal } \\
\text { hernia; oesophageal residue }\end{array}$ \\
\hline 2 & 1 & $\begin{array}{l}\text { Recurrent aspiration infections from } \\
\text { age } 10 \text { days ; episodes of vomiting and } \\
\text { restlessness at night; milk feeds only }\end{array}$ & Fluoroscopy & $\begin{array}{l}\text { Inefficient stripping action; hiatal } \\
\text { hernia; oesophageal residue }\end{array}$ \\
\hline 3 & 0.5 & $\begin{array}{l}\text { On three occasions sudden collapse } \\
\text { associated with white asphyxia and } \\
\text { subsequent aspiration pneumonia and } \\
\text { cyanosis }\end{array}$ & $\begin{array}{l}\text { Fluoroscopy } \\
\text { Cinefluorography }\end{array}$ & $\begin{array}{l}\text { Inefficient stripping action: hiatal } \\
\text { hernia with very free reflux; oesopha- } \\
\text { geal residue }\end{array}$ \\
\hline 4 & $1 \cdot 5$ & $\begin{array}{l}\text { Aspiration pneumonia; severe air } \\
\text { trapping-age } 6 \text { mths; later white } \\
\text { asphyxia, cardiac arrest, aspiration }\end{array}$ & $\begin{array}{l}\text { Cinefluorography on } \\
\text { two occasions }\end{array}$ & $\begin{array}{l}\text { Inefficient stripping action: Hiatal } \\
\text { hernia; oesophageal residue }\end{array}$ \\
\hline 5 & 4 & $\begin{array}{l}\text { pneumonia after swallowing solids } \\
\text { Recurrent episodes of choking and res- } \\
\text { piratory infection from age } 2 \text { yrs }\end{array}$ & Fluoroscopy & $\begin{array}{l}\text { Atonic below anastomosis; hiatal heinia } \\
\text { with very free reflux; oesophageal } \\
\text { residue }\end{array}$ \\
\hline 6 & 1 & $\begin{array}{l}\text { Recurrent respiratory infections; strained } \\
\text { food taken well but chokes and } \\
\text { heaves with lumpy food }\end{array}$ & Fluoroscopy & $\begin{array}{l}\text { Atonic below anastomosis; hiatal hernia; } \\
\text { oesophageal residue; aspiration of } \\
\text { contrast medium }\end{array}$ \\
\hline 7 & 5 & $\begin{array}{l}\text { Recurrent bronchitis; vomiting after } \\
\text { food; struggling and refusal to eat } \\
\text { solids until nearly age 2, thereafter }\end{array}$ & Fluoroscopy & $\begin{array}{l}\text { Atonic below anastomosis; hiatal hernia ; } \\
\text { oesophageal residue; prominent left } \\
\text { atrial impression }\end{array}$ \\
\hline 8 & $!$ & $\begin{array}{l}\text { episodes of choking and food sticking } \\
\text { Six episodes of aspiration pneumonia; } \\
\text { pools of saliva in throat; dribbles } \\
\text { from mouth; recurrent episodes of } \\
\text { "food sticking in throat" and vomiting; }\end{array}$ & $\begin{array}{l}\text { Fluoroscopy } \\
\text { Cinefluorography }\end{array}$ & $\begin{array}{l}\text { Atonic from pharynx to vestibule; } \\
\text { no hiatal hernia; oesophageal residue; } \\
\text { Prominent left atrial impression }\end{array}$ \\
\hline 9 & 2 & $\begin{array}{l}\text { still on mashed and strained food } \\
\text { Noisy cough for one year; lumpy food } \\
\text { sticks in throat }\end{array}$ & Fluoroscopy & $\begin{array}{l}\text { Yo-yo movements; absent stripping } \\
\text { wave; hiatal hernia-very free reflux; } \\
\text { oesophageal residue }\end{array}$ \\
\hline 10 & 10 & $\begin{array}{l}\text { Recurrent respiratory infections; in- } \\
\text { ability to take solids; food sticks in } \\
\text { throat; copious vomiting since birth- } \\
\text { severe symptom--described by mother } \\
\text { as "frightening" }\end{array}$ & Cinefluorography & $\begin{array}{l}\text { Yo-yo movements; absent stripping } \\
\text { wave; hiatal hernia; oesophageal } \\
\text { residue }\end{array}$ \\
\hline 11 & 2 & $\begin{array}{l}\text { Persistent cough; chronic wet chest; } \\
\text { frequent vomiting with mucus; at i } \\
\text { year, peanut stuck at lower end of } \\
\text { oesophagus-removed at oesophago- } \\
\text { scopy }\end{array}$ & Cinefluorography & $\begin{array}{l}\text { Yo-yo movements; absent stripping } \\
\text { wave; hiatal hernia; oesophageal } \\
\text { residue }\end{array}$ \\
\hline 12 & 2 & $\begin{array}{l}\text { Recurrent respiratory infections; still } \\
\text { on mashed foods }\end{array}$ & Fluoroscopy & $\begin{array}{l}\text { Yo-yo movements; absent stripping } \\
\text { wave; hiatal hernia; oesophageal } \\
\text { residue; prominent left main bronchial } \\
\text { impression }\end{array}$ \\
\hline 13 & $0 \cdot 2$ & $\begin{array}{l}\text { Aspiration pneumonia severe and per- } \\
\text { sisting }\end{array}$ & Cinefluorography & $\begin{array}{l}\text { Yo-yo movement resulting in pharyn- } \\
\text { geal filling even when erect; absent } \\
\text { stripping wave; no hiatal hernia; } \\
\text { oesophageal residue }\end{array}$ \\
\hline 14 & $0 \cdot 5$ & Severe aspiration pneumonia & $\begin{array}{l}\text { Cinefluorography } \\
\text { Fluoroscopy }\end{array}$ & $\begin{array}{l}\text { Yo-yo movement on one occasion; } \\
\text { no stripping wave visible; oesophageal } \\
\text { residue; hiatal hernia }\end{array}$ \\
\hline
\end{tabular}

No significant stricture, diaphragm, or fistula was present in any of the 14 patients.

Absence of normal effective stripping waves Pharyngeal deglutition was normal in all patients. Oesophageal stripping waves were absent in most patients, but in some indifferent, inefficient stripping waves were seen. However, common to all patients was the absence of normal efficient stripping waves, and in all an oesophageal residue following cessation of deglutition was present.

Four patients showed indifferent stripping action in the oesophagus. Initially stripping waves emptied the oesophagus, but later the waves were less efficient and an oesophageal residue remained (Fig. 1). The oesophagus showed little sign of contractility at this time.
Four patients had an oesophagus which showed lack of contractility. Sometimes a flicker of contractility was seen at the start of swallowing, but the oesophagus was subsequently completely atonic. The segment below the anastomosis was atonic in three (Fig. 2), and in one patient the oesophagus was atonic from the pharynx to the vestibule (Fig. 3).

Six patients exhibited the 'yo-yo phenomenon' (Fig. 4). Oesophageal stripping waves were absent and an oesophageal residue was present after the act or acts of deglutition had ceased. This oesophageal residue was associated with intermittent spontaneous contractions in the lower part of the oesophagus. With each contraction of the lower oesophagus, contrast medium was expelled into the upper oesophagus. Subsequently the con- 

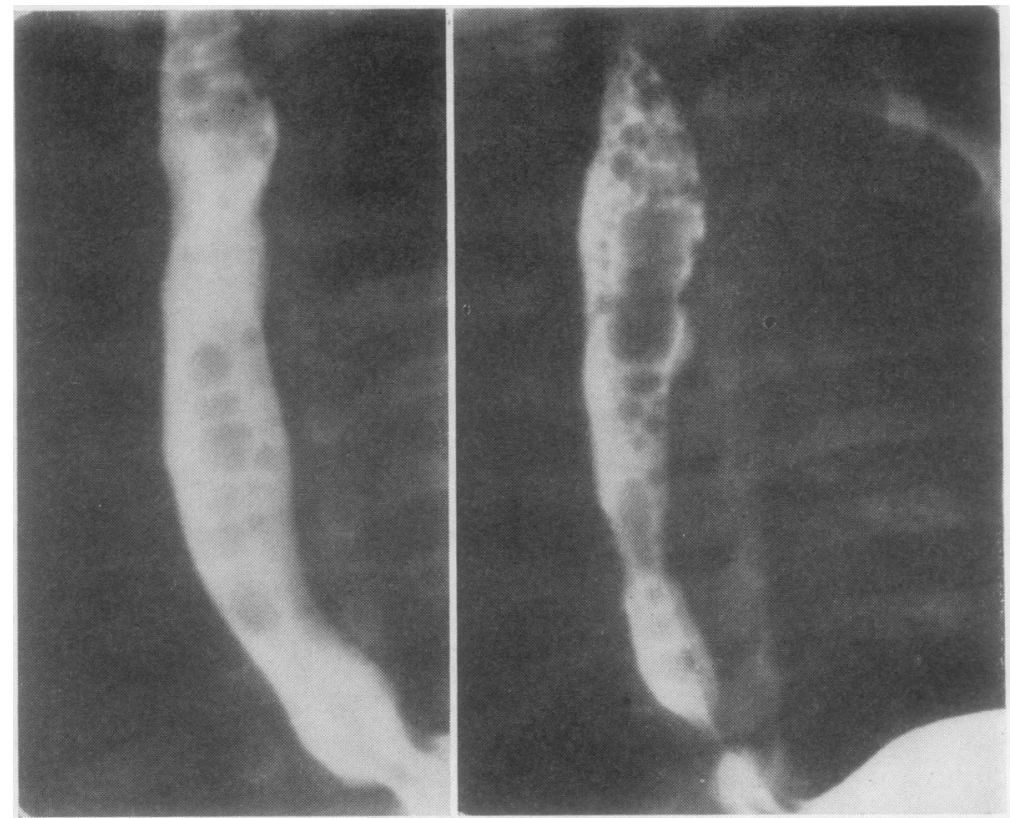

FIG. 1. Patient 2: (left) swallowing, vestibule open; (right) deglutition has ceased. Vestibule contracted; oseophageal residue because of inefficient stripping action; hiatal hernia.

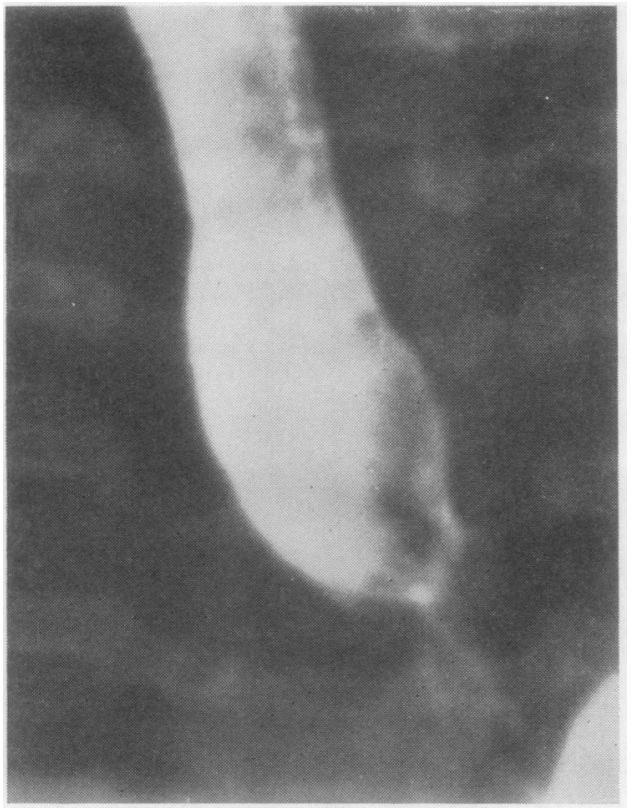

FIG. 2. Patient 6. About 2 min. after deglutition had ceased. Atonic oesophagus below anastomosis; vestibule closed; oesophageal residue; hiatal hernia. tracted segment relaxed, and contrast medium returned into the lower part of the oesophagus. This sequence, when repeated, resulted in continuing upward and downward movement of contrast medium, and this feature has become widely known as the yo-yo oesophagus. Even in the erect position the yo-yo movement resulted in reflux into the pharynx in one infant.

There was no evidence of contractility in the oesophagus above the anastomosis as a result of contrast medium entering it from below. Only in one patient did the yo-yo type contraction appear to interfere with flow down the oesophagus during deglutition, but this interference was transient.

Function of the vestibule Flow of contrast $N$ medium down the oesophagus, through the vesti- $\mathrm{N}$ bule, and into the stomach occurred as long as $\omega$ acts of deglutition continued. As deglutition ceased so flow through the vestibule ceased. Since oesophageal stripping action was defective a resi- $\mathbb{D}$ due remained in the oesophagus above the closed vestibule. In the erect position gravity-assisted emptying occurred, but this was incomplete (Fig. $3 d)$, especially in the younger patients. During deglutition the vestibule opened to the same extent $\varrho$ as is seen in normal patients. In achalasia the vestibule never opens in this way. 


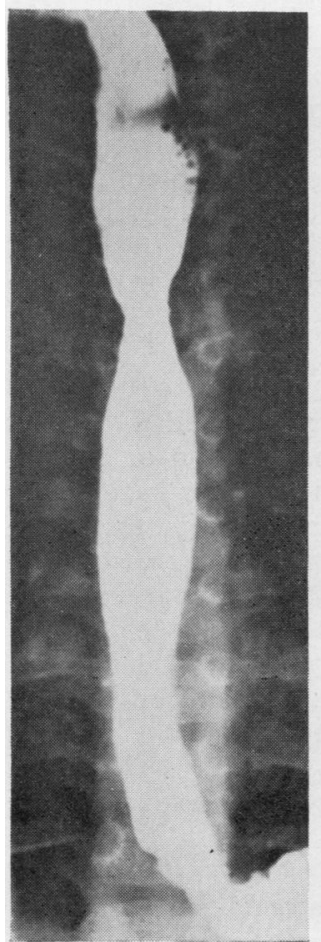

(a)

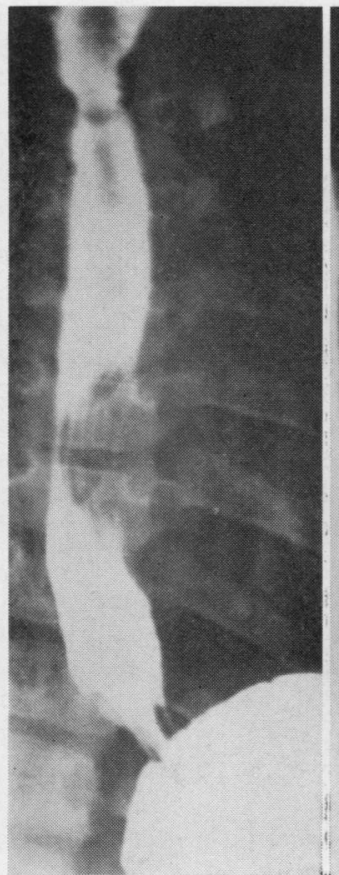

(b)

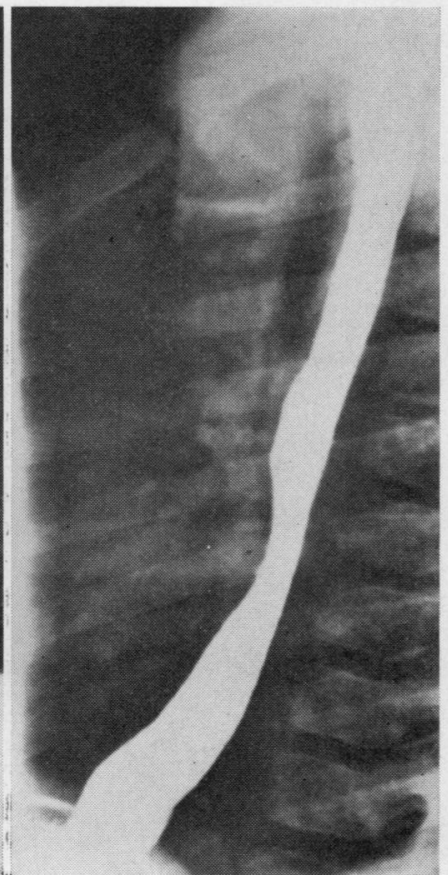

(c)

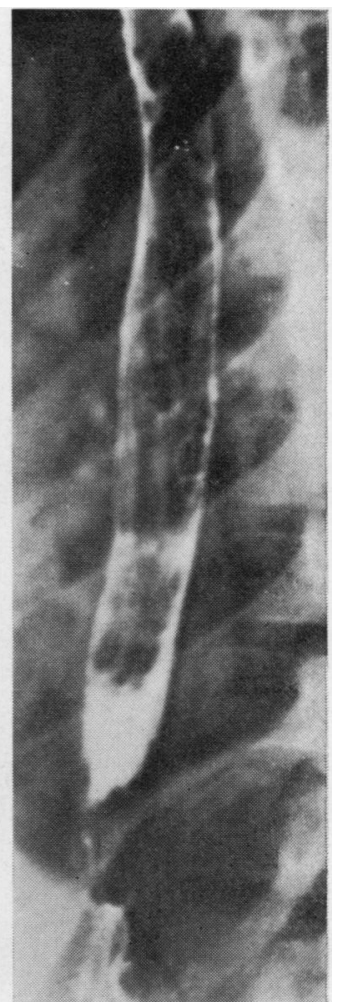

(d)

FIG. 3. Patient 8. Oesophagus atonic from pharynx to vestibule: (a) swallowing, vestibule open; (b) swallowing ceasing, vestibule starting to close, prominent left atrial impression; (c) prominent left atrial impression; (d) erect, some time after swallowing had ceased. Vestibule closed, atonic oesophagus containing air and barium.

Hiatal hernia In 12 of the 14 patients a sliding type of hiatal hernia was found. Reflux from the stomach into the oesophagus in the presence of a hiatal hernia is a well-recognized event, and it was seen in these patients.

Impressions of left main bronchus and left atrium These were unusually clearly seen in some patients because of the residue in the oesophagus (Figs 3 and 4).

CHEST RADIOGRaphS Pneumonic consolidation was seen in all patients. Repeated episodes of pulmonary consolidation were seen in some patients. All parts of the lung have been affected and there has been no predilection for the right upper lobe such as is seen in the patient with a tracheooesophageal fistula. In one patient (an infant aged 6 months) the pulmonary involvement was associated with marked air trapping and dilatation of the main pulmonary artery, suggesting pulmonary hypertension and cor pulmonale. Gradually, with treatment, the appearances returned to normal.

\section{DISCUSSION}

REVIEW OF PREVIOUS STUDIES Several observers have reported radiological studies of the oesophagus in patients in whom a technically successful repair of oesophageal atresia with closure of fistula had been achieved. Astley (1956) described 'the development of gastro-oesophageal incompetence due to operative traction on the cardia'. Haight (1957) noted that in five patients the normal oesophageal contraction or stripping wave failed to pass through the site of anastomosis, but it reappeared in the lower oesophageal segment below the anastomosis. Kirkpatrick, Cresson, and Pilling (1961) conducted cineradiographic studies on 15 patients who had developed dysphagia, impaction of foreign bodies, and aspiration pneumonia. Contrast medium remained above the anastomosis until a mass contraction propelled a major part of the bolus past the line of anastomosis. Subsequently, after contrast medium had reached the lower oesophagus, contraction in that part of the oesophagus resulted in retrograde but 


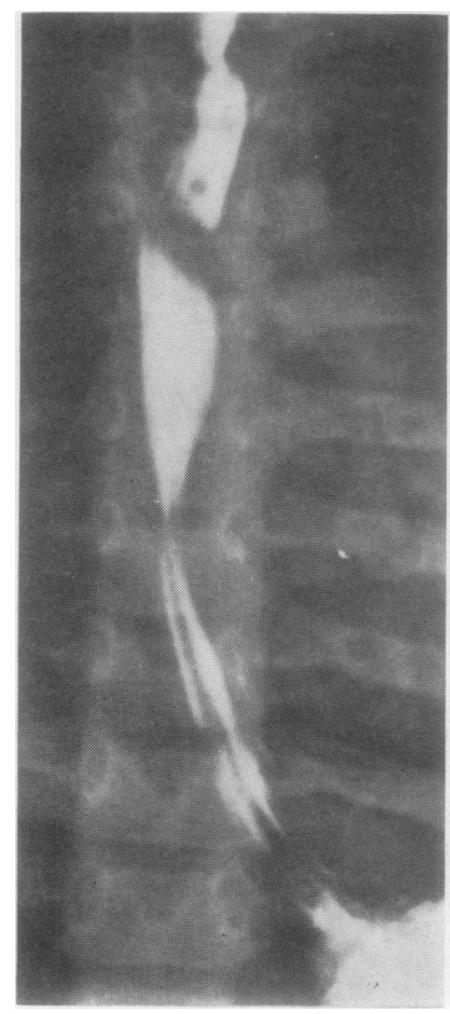

FIG. 4. Patient 12. Deglutition has ceased. Oesophageal residue. Lower oesophagus contracts, giving upward phase of yo-yo movement. Prominent left main bronchus impression.

also some antegrade flow of the contrast medium. Studies on 49 patients, all of whom exhibited a yo-yo phenomenon, were reported by Desjardins, Stephens, and Moes (1964). Six of the 49 often had difficulty with swallowing and had recurrent respiratory infections. Girdany (1963) has pointed out that the yo-yo phenomenon is not a feature specific to the patient who has had a repair of oesophageal atresia.

\section{INTERPRETATION OF RADIOLOGICAL FINDINGS}

Evidence favouring an inherent oesophageal abnormality All patients showed an absence of normal efficient stripping waves with failure to empty the oesophagus when deglutition ceased. Kirkpatrick et al. (1961) examined a patient with tracheo-oesophageal fistula and oesophageal stenosis pre-operatively and found an absence of stripping waves below the stenosis. At the Hospital for Sick Children cinefluorographic studies were carried out pre-operatively on one patient aged 5 years and on another aged 11 years in the prone-oblique position during normal deglutition. A tracheo-oesophageal fistula (confirmed at operation) was shown in each patient, but no stripping wave above or below the fistula was seen. These findings suggest that the absence of normal stripping waves can be an inherent feature of the patient with a tracheo-oesophageal fistula. Maybe this is also the case when an atresia has been present.

Effect of surgical technique It seems unlikely that surgical techniques are related either to the development of the yo-yo phenomenon or to the atonic oesophagus. Five surgical teams have been concerned with the initial operation in these 14 patients. In some patients meticulous care has been taken to achieve primary anastomosis with the minimum of dissection above and below the site of anastomosis. In others it has been necessary to carry out dissection above and below the anastomosis to produce a sound union.

\section{RELATION OF RADIOLOGICAL FINDINGS TO SYMPTOMATOLOGY}

Abnormal antegrade oesophageal flow (Fig. 5) Factors affecting flow down the oesophagus in these 14 patients are (1) inefficient oesophageal

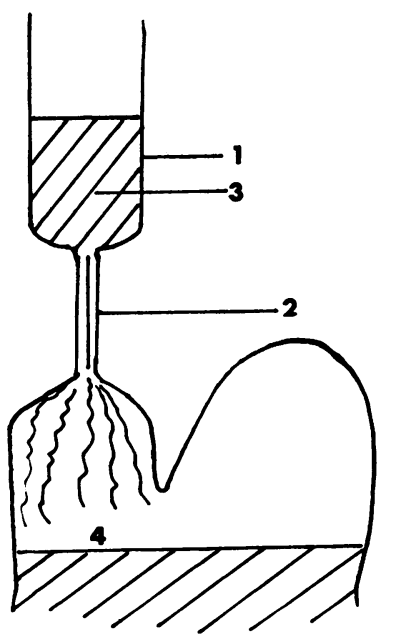

FIG. 5. Abnormal antegrade oesophageal flow. Patient erect. Inefficient or absent stripping wave in the wall of the oesophagus (1) and closure of vestibule (2) on cessation of deglutition result in failure of oesophageal content (3) to pass into stomach (4). 
contractility; (2) gravity-in the erect position this is important, especially when liquids (notably high density mixtures such as barium) are being swallowed; (3) repeated acts of deglutition in which repeated pharyngeal contractions propel material into and down the oesophagus ; (4) vestibular closure after cessation of deglutition which with (1) results in an oesophageal residue.

In infants in whom sucking and pharyngeal deglutition are normal, intake into the abnormal oesophagus may exceed output through the vestibule into the stomach. This imbalance would account for the episodes of choking in this age group.

In older children episodes of choking are less common because they have a larger capacity oesophagus of greater length, feeding occurs in the upright posture, and intake is personally controlled. Dysphagia with solid foods is then the dominant symptom. Defective oesophageal stripping action fails to impel poorly chewed, solid, lumpy food down the oesophagus into the stomach. In the erect position, low density food, such as peanuts, may linger in an oesophageal fluid residue and may impact in the vestibule.

Retrograde oesophageal flow (Fig. 6) The presence of a hiatal hernia impairs refluxpreventing mechanisms at the oesophago-gastric junction. Once reflux has occurred, defective oesophageal stripping action can result in unimpeded flow up the oesophagus as far as the pharyngeal sphincter. The yo-yo movement also results in retrograde oesophageal flow to this level. Horizontal beam cinefluorographic studies with the infant swallowing supine have shown that the fluid level in the stomach, after even a small feed, is considerably higher than the vestibule, oesophagus, pharynx, and larynx. The young infant spends much of its life lying on its back ; in this position only the pharyngeal sphincters prevent reflux into the pharynx and vomiting.

Aspiration pneumonia Abnormalities of transit down the oesophagus, reflux with unimpeded retrograde flow through the oesophagus, the yo-yo movement in some patients, and an oesophageal residue after deglutition may all account for aspiration into the respiratory tract.

\section{MANAGEMENT}

Erect position In young children this results in gravity-assisted oesophageal emptying, and it diminishes the chance of reflux.

Thickened feeds In infancy these reduce the risk of regurgitation and slow the rate of feeding, thereby avoiding imbalance between the rate of intake into the oesophagus and the rate of oesophageal emptying into the stomach.

Water at the end of a feed Theoretically a little water given at the end of each feed should

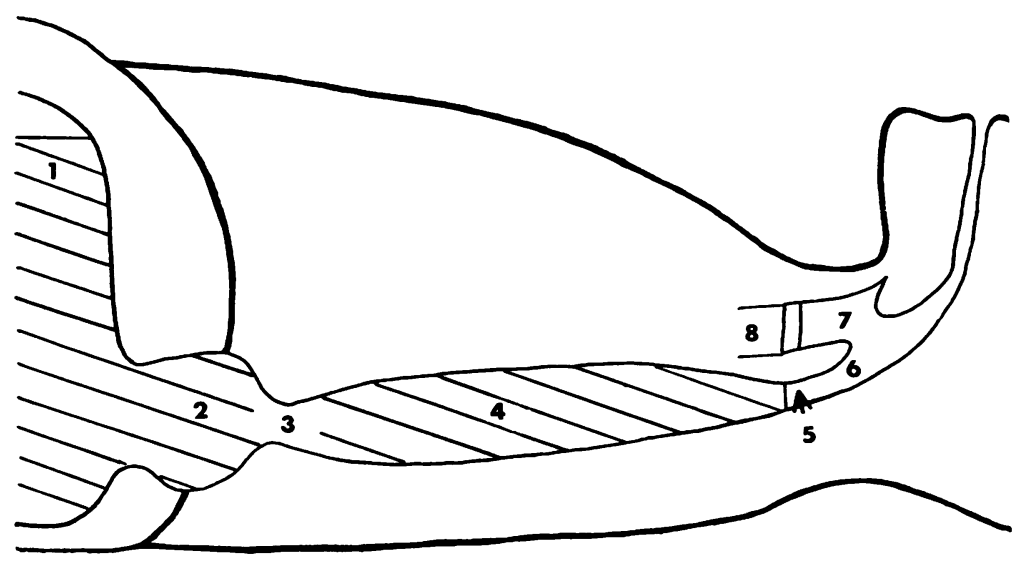

FIG. 6. Retrograde oesophageal flow. Diagrammatic representation based on cine film using horizontal $x$-ray beam with patient lying supine. Fluid level in stomach (1) is higher than hiatal hernia (2) and vestibule (3), thus permitting reflux from stomach into oesophagus (4). The oesophagus has an inefficient secontary stripping wave. Flow up the oesophagus occurs unimpeded to the lower pharyngeal sphincter (5). When this sphincter relaxes on deglutition the oesophageal content can pass into the pharynx (6), larynx (7), and trachea (8), all of which are lower than the level in the stomach (1). 
result in a residue of water in the oesophagus rather than a residue of milk or food.

Older children Dysphagia is the major problem in older children, and this may be lessened by giving minced food and/or water between mouthfuls of well-chewed solid food. The vestibule opens with each act of deglutition, and the water provides an intermittent oesophageal lavage.

\section{SUMMARY}

Fourteen patients who had previously had a technically successful repair of oesophageal atresia and fistula presented with episodic aspiration pneumonia and dysphagia.

Conventional fluoroscopy and spot-filming and cinefluorographic studies of oesophageal function were carried out. Significant findings in the oesophagus were (1) inefficient stripping action ; (2) oesophageal residue after deglutition; (3) sliding hiatal hernia with reflux. Chest radiographs showed evidence of aspiration pneumonia. One patient had severe air trapping and probably pulmonary hypertension.

Correlation of symptomatology with radiological findings suggests that the abnormality of oesophageal function is causative. Abnormalities of transit down the oesophagus, reflux through the oesophagus, and the oesophageal residue after deglutition can account for choking, dysphagia, vomiting, and aspiration into the respiratory tract.

Knowledge of these oesophageal abnormalities provides a logical approach to management. In infancy the erect position, thickening of feeds, and a swallow of water at the end of the feed can all be helpful. Dysphagia in older children can be ameliorated by the swallowing of water between mouthfuls of well-chewed or minced food.

We wish to thank Professor A. W. Wilkinson, Mr. G. H. Macnab, Mr. H. H. Nixon, and Mr. J. D. Atwell, who have undertaken the care of some of the patients in this series. We are indebted to Dr. R. E. Bonham-Carter, of the Thoracic Unit, for a considerable amount of help and advice. We must thank Dr. L. G. Blair for his helpful comments on the preparation and presentation of the paper.

\section{REFERENCES}

Astley, R. (1956). Radiology of the Alimentary Tract in Infancy, p. 7. Arnold, London.

Desjardins, J. G., Stephens, C. A., and Moes, C. A. F. (1964). Results of surgical treatment of congenital tracheo-esophageal fistula with a note on cine-fluorographic findings. Ann. Surg., 160, 141.

Girdany, B. R. (1963). The esophagus in infancy: congenital and acquired diseases. Radiol. Clin. N. Amer., 1, 557.

Haight, C. (1957). Some observations on esophageal atresias and tracheo-esophageal fistulas of congenital origin. J. thorac. Surg., 34, 141.

Kirkpatrick, J. A., Cresson, S. L., and Pilling, G. P. (1961). The motor activity of the esophagus in association with esophageal atresia and tracheoesophageal fistula. Amer. J. Roentgenol. atresia. 884 .

Waterston, D. J., Bonham-Carter, R. E., and Aberdeen, E. (1963). Congenital tracheo-oesophageal fistula in association with oesophageal atresia. Lancet, 2, 55. 